\title{
Transport infrastructure and services: an Asia and developing world
}

\author{
Toshiyuki Yamamoto $\cdot$ Antti Talvitie
}

Published online: 18 September 2011

(C) Springer Science+Business Media, LLC. 2011

Infrastructure is a fundamental factor in the development of countries and regions. Both the quality and the quantity of the infrastructure have significant effects on productivity. In particular, good transport infrastructure is essential for regional cooperation and integration, helping lagged regions catch up with the developed regions. Several Asian countries and regions have shown significant developments over the past several decades. Rapid infrastructure investments have supported that development and economic growth. However, there is evidence that development gaps between Asian countries have widened over time. Therefore, the transport infrastructure investments in Asian countries and regions merit investigation in terms of quality, quantity, benefits and costs, and benefit transfers to promote efficient and fair infrastructure investments for the future.

This Special Issue of Transportation consists of five papers submitted to Transportation and two papers selected from the presentations at the 12th World Conference on Transport Research, held at Lisbon, Portugal, in 2010. The former group of papers addresses several issues of Asian transport infrastructure while the latter is not limited to Asia but is generally applicable in developing countries. The latter group of papers was presented in the conference in Topic Area H: Transport in developing countries, which deals with all the transport issues in the developing and transition countries. The shared interests in the papers relate to all modes of transport infrastructure and development and management of that process.

Hong et al. investigate transport infrastructure and regional economic growth in China. China's economy has grown rapidly in the past decade, and the divergence in the development between regions has become obvious, and of concern. They examine the effects of various types of transport infrastructures, including land, air and water, using a panel data from 1998 to 2007. The results suggest that both land transport and water transport have a significant impact on economic growth, while the effect of the air transport is weak.

T. Yamamoto $(\bowtie)$

Nagoya University, Nagoya, Japan

e-mail: yamamoto@civil.nagoya-u.ac.jp

A. Talvitie

Aalto University, Helsinki, Finland

e-mail: antti.talvitie@aalto.fi 
The authors note that the development of land transport infrastructure contributes more to economic growth in regions with poor land transport conditions.

Land transport includes both the roads and the railways. Utsunomiya and Hodota evaluate railways, especially the high speed train networks connecting regions within each Asian country. They examine the high speed train networks in Japan, Taiwan, Korea and China from the financial view point. The authors note that a high speed rail systems are barely financially viable even in areas with high population density, and that the government needs to be transparent in showing the cost/revenue comparisons with other transport modes.

Road networks experience congestion especially in large metropolitan regions owing to rapid motorization which has accompanied the economic growth, leading to deteriorating service quality. In order to reduce the excess demand, Singapore was the first to implement congestion pricing in 1975. Congestion pricing is a market mechanism to approach the optimum level of the demand. The optimum toll can be determined theoretically if origindestination demand functions are known. In reality such information is difficult to obtain. Meng and Liu tackle this problem by trial-and-error to derive the effective toll pattern. They propose a minimization model for a constrained probit-based SUE (stochastic user equilibrium) problem, and show the effectiveness of the model in practical situations. The model is tested using the network data of the Singapore electric road pricing (ERP) system on the downtown Orchard Road. The results numerically verify the convergence of the proposed method to the effective toll while the method currently used for actual ERP system is shown to fail to converge.

Traffic congestion also adversely affects the level of service of bus operations. The decreased level of service reduces the bus ridership, resulting in reduced bus services. This vicious cycle of decline has been experienced in many metropolitan areas. In order to improve the quality of service, Seoul-among other Asian cities-restructured the bus service network in 2004. Kim et al. evaluate the performance of the new system. Salient features of the restructuring are semi-public operation, an integrated public transport fare system, median bus lanes and a bus management system. The performance of the comprehensive reform is analyzed before and after the changes. They report an improvement $30-75 \%$ in average bus speeds and attribute it mainly to the median bus lane system. They also report a $17 \%$ increase in bus ridership. The authors note that the number of subway passengers also increased due to the integrated smart card fare system.

A transport network not only supports everyday cooperation and integration of the affected regions, but it also mitigates the effects of disasters. When the Tohoku Region Pacific Coast Earthquake struck Japan in March 2011, 'Operation COMB' was implemented where a "comb" shaped emergency route was placed to open traffic to the more affected areas from the tsunami. To implement such an operation, it is critical to analyze the vulnerability of the network to identify the vulnerable links, and then strengthen those critical links or add redundancy into the network. Luathep et al. propose a practical method for vulnerability analysis, and investigate the vulnerability of the road network of Bangkok metropolitan area. Conventional methods for vulnerability analysis entail a significant computational burden, which has been recognized as the most challenging issue for applications to large-scale real networks. The authors propose a sensitivity-based approach to improve computational efficiency, and show the efficiency of the approach using Bangkok metropolitan area network, which has over 3,000 links. Conventional approaches need the same number of calculations as the number of links. The computation time is substantially reduced by the proposed approach. Links on the outskirts of the area serving high travel demands, links serving high demand induced by tourism demands, and links 
connecting bridges across the main river are identified as the most vulnerable in the network.

The promotion of bicycling has recently become part of urban transport strategy. However, most Asian cities and cities in developing countries do not have a significant length of dedicated bicycle lanes or pathways, and the bicyclists share the road with motor vehicles, affecting the quality and safety of bicycling. Providelo and Sanches evaluate the quality of roads for bicycling in urban areas in several Brazilian cities. Fourteen characteristics were identified in prior focus group discussions including motor vehicle traffic volume, motor vehicle speed, and presence of heavy vehicles. The 14 attributes are used in an attitude survey to assess perceptions about them. The results indicate that the most important characteristics of roads for positive bicycling experience are the lane width and motor vehicle speed. The authors note that bicyclists are willing to share the road with cars if the cars have low speeds and the lanes are wide.

The last paper of this special issue by Gogelia and Talvitie discusses transport project and program development in the developing countries from the managerial and technical view points. Transport projects funded by international financing institutions are considered. The authors attend to the details of project implementation in the frame of project development objectives and discuss the often repetitive issues based on several projects in many countries over two decades. The aid details and important issues are addressed in a practical manner. According to the authors the 'devil is in the details' whose resolution significantly influences the quality of program/project outcomes. Good solutions are likely to be found from the stories on successes and failures alike, which should guide the theory and technique of the development process.

The seven papers in this special issue are indicative of the wide range of issues and problems present in the development and provision of transport infrastructure and services in Asia and the developing world. The papers in this Special Issue focus on different kinds of transport problems and approaches to solving them. Yet the case study countries, China, Japan, Singapore, Korea, Thailand and Brazil, also share them commonly. The studies and experiences in these countries are worth sharing as Gogelia and Talvitie encourage 'story-telling' as one part of managing the technical process of development experience.

\section{Author Biographies}

Toshiyuki Yamamoto is a Professor in EcoTopia Science Institute at Nagoya University, Japan. His research interests include household vehicle ownership and use, car sharing, intelligent transport system utilizing probe vehicles, traffic safety and travel behavior in general. He is an Editor of Transportation.

Antti Talvitie is currently in private practice. Previously he worked in the World Bank, in the private sector, served as Director of Highway Construction and Maintenance in Finland, and as Professor and Chairman of Civil Engineering at the State University of New York in Buffalo. He has PhD from Northwestern University, Evanston, IL, and Certificate in Psychoanalysis from the Boston Graduate School in Psychoanalysis. 